\title{
Metabolic Syndrome, Chronic Kidney, and Cardiovascular Diseases: Role of Adipokines
}

\author{
Manfredi Tesauro, ${ }^{1,2}$ Maria Paola Canale, ${ }^{1}$ Giuseppe Rodia, ${ }^{1}$ Nicola Di Daniele, ${ }^{1}$ \\ Davide Lauro, ${ }^{1}$ Angelo Scuteri, ${ }^{3}$ and Carmine Cardillo ${ }^{4}$ \\ ${ }^{1}$ Department of Medicina Interna, Università di Tor Vergata, Viale Oxford 81, 00133 Rome, Italy \\ ${ }^{2}$ Laboratory of Molecular Medicine, Department of Internal Medicine, University of Rome Tor Vergata, \\ Viale Oxford 81, 00133 Rome, Italy \\ ${ }^{3}$ UOC Geriatria INRCA, POR Roma, 00189 Rome, Italy \\ ${ }^{4}$ Università Cattolica del Sacro Cuore, 00168 Rome, Italy \\ Correspondence should be addressed to Manfredi Tesauro, mtesauro@tiscali.it
}

Received 1 October 2010; Revised 30 November 2010; Accepted 7 January 2011

Academic Editor: Ken Ichi Aihara

Copyright (C) 2011 Manfredi Tesauro et al. This is an open access article distributed under the Creative Commons Attribution License, which permits unrestricted use, distribution, and reproduction in any medium, provided the original work is properly cited.

\begin{abstract}
Obesity is a chronic disease, whose incidence is alarmingly growing. It is associated with metabolic abnormalities and cardiovascular complications. These complications are clustered in the metabolic syndrome (MetS) leading to high cardiovascular morbidity and mortality. Obesity predisposes to diabetic nephropathy, hypertensive nephrosclerosis, and focal and segmental glomerular sclerosis and represents an independent risk factor for the development and progression of chronic kidney disease (CKD). Albuminuria is a major risk factor for cardiovascular diseases (CVDs). Microalbuminuria has been described as early manifestation of MetS-associated kidney damage and diabetic nephropathy. Obesity and MetS affect renal physiology and metabolism through mechanisms which include altered levels of adipokines such as leptin and adiponectin, oxidative stress, and inflammation. Secretory products of adipose tissue also deeply and negatively influence endothelial function. A better understanding of these interactions will help in designing more effective treatments aimed to protect both renal and cardiovascular systems.
\end{abstract}

\section{Introduction}

The prevalence of obesity, among both adults and children, has worldwide increased over the past two decades; a phenomenon which is predominantly attributed to the change in dietary habits and lifestyle modifications $[1,2]$. It is clearly knows that central obesity is an independent risk factor for CVD and is associated with Mets. Insulin resistance (IR) is a key feature of the Mets and consists in a decreased sensitivity or responsiveness of peripheral tissues to the metabolic action of insulin [3]. IR as well as all components of the Mets are associated with altered functions of endothelium which lead to CVD [4]. Hyperlipemia and coronary artery disease are also consequences of obesity which through a cascade of various reactions lead to kidney dysfunction. Moreover, obesity-induced sleep apnoea activates sympathetic nervous system increasing the tone of the glomerular efferent arterioles and the secretion of renin and angiotensin [5]. In the last decade, obesity has been suggested as a risk factor for chronic kidney disease, decline of renal function, and lowgrade albuminuria, but conflicting results have been reported [6-10]. Yet obesity, per se, even in the absence of the abovementioned medical complications alters renal physiology and metabolism. In this paper, we will examine the role of adipose tissue-secreted factors and the mechanisms of obesity-induced renal and vascular injury leading to chronic kidney and cardiovascular diseases.

\section{Epidemiology of Mets, CKD, and CVD}

CVD accounts for premature death in about $50 \%$ of dialysis patients [11]. As early as 1974, Lindner et al. demonstrated that dialysis patients have a higher prevalence of CVD compared to the general population [12]. The strong association between mild CKD and CVD has been shown, and recently Henry et al. reported that mild to moderate CKD is strongly 
associated with an increase in cardiovascular mortality [13, $14]$.

Ninomiya et al. recently examined the relationship between Mets and CKD [15]. They performed a slope analysis of the association between the glomerular filtration rate (GFR) slope and Mets by using a multiple regression model. GFR decreased significantly faster in patients with 4 or more Mets components compared with those who had 1 or no components. Moreover the mean of the GFR slope was significantly lower in subjects with 3 Mets components in the 60 year and over group. In a large cohort of the NHANES III study with baseline normal renal function, Chen et al. examined the risk of developing CVD following patients for more than 20 years [16].

Interestingly, there also was a 2 -fold increase in the risk of microalbuminuria that correlates with the number of components of Mets. Moreover, even low-grade albuminuria below the conventional cut-off point for albuminuria was associated with increased prevalence of CKD [17]. Data from the MESA [18] and LIFE studies [19] clearly demonstrated that albuminuria is one of the strongest risk factors for cardiovascular disease (CVD).

\section{Adipose Tissue As an Active Endocrine Organ}

There are multiple changes in adipose tissue in obesity including increase in numbers and size of adipocytes, infiltration of adipose by mononuclear cells, rarefaction of blood vessels, increases in adipocyte turnover rate, differentiation, and apoptosis [20]. The capillary diffusion capacity is reduced in patients with obesity and, in contrast with the response of lean subject, the adipose blood flow does not increase in response to food in obese patients [21]. Since the discovery of leptin as an adipocyte-derived satiety factor, adipose tissue is increasingly being considered as an endocrine organ. Adipose tissue secretes into the circulation a number of proteins and nonprotein factors that regulate glucose and lipid metabolism throughout the body. Among these bioactive adipokines, only adiponectin (ADN), leptin, adipsin, and visfatin are almost synthesized exclusively by adipocytes. In obese patients, the production of ADN is reduced [2224]. ADN is a $30 \mathrm{kDa}$ protein present as oligomers in the blood stream and has insulin-sensitizing, antiatherogenicity, and anti-inflammatory properties. Reduction of ADN levels is a consistent feature among obese patients who have evidence of IR and often develop diabetes mellitus. ADN plasma levels also negatively correlate with coronary artery disease and dyslipidemia in both mice and humans [25-28]. Overexpression or AND administration reduces oxidative stress, inflammation, IR, and vascular damage. Becker et al. found that low ADN levels in mild to moderate kidney disease were correlated with cardiovascular events [29]. The potential link between ADN levels and low-grade albuminuria was first observed in a clinical study where essential hypertensive patients had a negative correlation between ADN levels and low-grade albuminuria [30]. Later on, similar results were also obtained in obese patients from different ethnic groups [31, 32]. Despite some controversial observations [33, 34], clinical data strongly suggest the potential causative role of $\mathrm{ADN}$ in the development of albuminuria in obese patients. An important contribution in understanding the potential link between obesity and kidney damage comes from the work by Sharma [32]. In a recent study, he showed that ADN knockout mice had baseline increased albuminuria (twice normal values) with podocyte foot process effacement. Morphologically, the endothelium appeared to be normal under electron microscopy. Podocytes expressed the AdipoR1 receptor and $\mathrm{ADN}$ regulated an isoform of NAPDH oxidase through the AMPK pathway [32]. When treating ADN knockout mice with ADN, proteinuria was reversed and foot processes were normalized. In mice, Sharma et al. [32] also demonstrated that ADN deficiency was a susceptibility factor for early diabetic kidney disease. Consequently, the podocyte may play an important role in determining albuminuria associated with obesity [35]. The role of endothelial dysfunction may be important for albuminuria as well. At the present time, no data are available to document the presence of glomerular endothelial dysfunction in the presence of microalbuminuria. On the other hand, it has been established that podocyte dysfunction contributes to endothelial dysfunction $[36,37]$.

The increase of visceral fat promotes synthesis of proinflammatory adipokines which cause tissue-specific increase in reactive oxygen species derived from NADPH oxidase. Adipose tissue oxidative stress results in the development of systemic oxidative stress and inflammation, which further lead to the development of metabolic abnormalities. Leptin, a 167 amino acid polypeptide, is expressed mainly by adipocytes; leptin concentration positively correlates with adiposity [38], and hyperleptinemia is an independent risk factor for coronary artery disease [39] and a strong predictor of acute myocardial infarction [40]. Additionally, leptin has been implicated in many atherogenic processes, including platelet aggregation and thrombosis [41-43]; production of inflammatory cytokines, for example, TNF- $\alpha$, IL-6, and IL12 [44]; calcification of vascular smooth muscle cells [45]. Interestingly, recent reports have demonstrated that leptin possesses cytokine-like properties and that elevated plasma leptin levels occur concomitantly with elevated IL-6 and Creactive protein in human obesity, the Mets, and noninsulindependent diabetes mellitus [46-48]. Animal testing showed that leptin induces proliferation of glomerular endothelial cells, enhances glomerular TGF- $\beta 1$ beta expression, and increases collagen type IV mRNA production [49]. These factors result in focal glomerulosclerosis, glomerular and mesangial glucose uptake, and proteinuria [49]. Additionally, leptin is also associated with adrenergic activation, increased blood pressure and tachicardia, contributing to obesityrelated hypertension and kidney damage [50-53].

In recent years, a great deal of attention has been focused on the orexigenic peptide ghrelin which is predominantly secreted by the stomach $[54,55]$. Patients with obesityrelated Mets have reduced ghrelin circulating levels. Ghrelin has important vascular actions; it acutely stimulates production of NO in vascular endothelium through a PI3-kinasedependent mechanism involving phosphorylation of Akt which directly phosphorylates and activates eNOS leading to increased production of NO [56]. This signaling pathway 
is similar to that used by insulin to promote increased production of NO in vascular endothelium. Moreover, we have demonstrated that intra-arterial ghrelin administration acutely improves endothelial dysfunction by increasing nitric oxide (NO) and decreases ET-1-dependent vasoconstriction, thereby restoring the physiological balance between these opposing vascular mediators in patients with central obesity $[57,58]$.

Thus, therapeutic interventions including weight loss, exercise, or pharmacological therapies that increase plasma ghrelin levels could to contribute to this strategies by mimicking and/or augmenting beneficial effects of increased insulin sensitivity.

Dysregulation of adipokines synthesis and release into the blood stream occur in obese patients and play a critical role in promoting IR [59-61]; diabetes and dyslipidemia are characterized by low adiponectin levels and elevated levels of inflammatory adipokines such as TNF-alpha (TNF- $\alpha$ ) [62].

Adipose tissue also expresses a local renin-angiotensin system (RAS). Adipocytes express RAS receptors and synthesize and secrete angiotensinogen and angiotensin peptides [63]. RAS is a hormonal cascade that governs vascular tone, fluid-electrolyte balance, and blood pressure [64].

\section{Adipokines and Cardiac Function}

Many studies have shown effects on the heart of various adipokines. TNF- $\alpha$ has been considered to be a critical factor in the pathogenesis of cardiac contractile dysfunction and heart failure. Transgenic mice with overexpression of TNF- $\alpha$ develop severe dilated cardiomyopathy [11], and TNF- $\alpha$ directly depresses cardiomyocyte contractility and induces apoptosis of cardiomyocytes in vitro [65]. TNF- $\alpha$ has negative inotropic effects on cardiomyocytes in vitro, and leads to heart failure in mice [66-72]. In addition, elevated serum TNF- $\alpha$ levels have been associated with the progression of heart failure in patients [66].

Experimental findings have shown that adiponectin has several beneficial effects in the cardiovascular system. Adiponectin plays an essential role in the maintenance of heart architecture, as the cytokine may attenuate angiotensin II-induced cardiac hypertrophy [73] and attenuate cardiomyocyte contractile dysfunction in $d b / d b$ diabetic obese mice via a mechanism possibly related to c-Jun and IRS-1 phosphorylation [74].

Moreover, adiponectin represses atherosclerotic lesions in a mouse model of atherosclerosis, and adiponectindeficient mice exhibit an accelerated vascular remodeling response to injury [75]. In addition, adiponectin stimulates nitric oxide production in endothelial cells through AMPKdependent and AMPK-independent phosphorylation of endothelial nitric oxide synthase (eNOS) [76, 77] and hypoadiponectinemia is associated with the progression of left ventricular hypertrophy (LVH), which is accompanied by diastolic dysfunction [78].

An association between serum leptin concentrations and various cardiovascular risks, including myocardial infarction [79], coronary heart disease [40], stroke [39], chronic heart failure [80], and left cardiac hypertrophy [81], has been observed.

Several works suggested that leptin could be an important link between obesity and development of cardiovascular disease [82]. This might be mediated through various effects of leptin including effect on blood pressure [83], inflammatory vascular response $[42,84]$, and platelet aggregation $[85$, 86]. High levels of leptin are associated with lower arterial distensibility [87] and have also been shown by several investigators to promote angiogenesis, enhance the calcification of vascular cells, and potentiate the prothrombotic platelet aggregation $[86,88]$ moreover, obese individuals possess higher plasma levels of prothrombotic factors such as fibrinogen, von Willebrand factor, factor VII, and plasminogen activator inhibitor-1 (PAI-1), which lead to a higher risk of thrombosis and atherosclerosis. The levels of pro-thrombotic factors such as fibrinogen, von Willebrand factor, factor VII, and plasminogen activator inhibitor-1 (PAI-1) are shown to be directly correlated with leptin levels [89].

Ghrelin is not produced by the adipose tissue; however, the functions of this gastric peptide are closely related to those of adipokine regarding metabolic and cardiovascular function.

Ghrelin has been demonstrated to have cardiovascular effects, both in animals and humans. Ghrelin protects the heart from ischemia in rats, and chronic administration of the peptide improves cardiac contractility in animals with chronic heart failure [90]. Furthermore, in the heart of rats subjected to ischemia followed by reperfusion, ghrelin reduces the infarct size [91], increases cardiac output, diastolic thickness of the noninfarcted wall, and attenuates the development of cardiac cachexia in rats with heart failure [92]. In humans, ghrelin administration reduces cardiac afterload and increases cardiac output and systemic vascular resistance, without changing heart rate [93]. Moreover, ghrelin administration increases exercise capacity and improves left ventricular function and muscle wasting in patients with chronic heart failure [94-96].

\section{Adipose Tissue Inflammation}

Recent observations from Kamei et al. [97] on transgenic mice have led to the concept that obesity contributes to IR and diabetes promoting a condition of chronic, low-grade inflammation of the adipose tissue because of additional infiltration and accumulation of inflammatory macrophages [98-102]. The macrophage content of adipose tissue is higher in visceral obesity compared to subcutaneous obesity, leading to the concept that visceral fat more than subcutaneous one plays a central role in the development of IR [103]. Macrophages appear to be recruited from the circulation, and adipocyte-derived factors might be involved in this process.

Monocyte chemoattractant protein-1 (MCP-1) is produced predominantly by macrophages and endothelial cells and is considered one of the most important potent chemotactic factors for monocytes. The increase of MCP-1 expression in adipose tissue contributes to the macrophage infiltration and IR associated with obesity. 
The general low-grade chronic inflammatory state, closely related to obesity, may affect insulin action by suppressing insulin receptor signalling via serine phosphorylation of insulin receptor substrates at metabolically relevant sites [104]. Because favourable metabolic and hemodynamic actions of insulin share common intracellular transduction pathways [105], inflammation may simultaneously contribute to both IR and vascular dysfunction, two cardinal and interrelated features of the Mets. Moreover, macrophages become activated and secrete inflammatory cytokines such as TNF-alpha and IL.6 which in turn decrease insulin action on adipocytes, determine hypoadiponectin, and increase leptin production $[98,106]$. TNF- $\alpha$ indeed plays a central role in the pathophysiology of IR and vascular damage in patients with obesity; the deletion of the TNF- $\alpha$ gene or the TNF receptors has proven effective to improve insulin action in both genetic and dietary models of rodent obesity. Overexpression of TNF- $\alpha$ has previously been reported in obese adipose tissue $[62,107]$, as well as in the skeletal muscle of insulin-resistant animals and humans and exposure to TNF- $\alpha$ acutely inhibits insulin-stimulated glucose uptake. Additionally, TNF- $\alpha$-induced vasculopathy is characterized by increased vascular reactive oxygen species.

We have previously demonstrated that TNF- $\alpha$ neutralization with the monoclonal antibody infliximab ameliorates insulin-stimulated vascular reactivity in Mets [108]. Our findings suggest that increased oxidative stress is involved in mediating the effects of TNF- $\alpha$ on insulin-stimulated vasodilator capacity. The beneficial action on vascular reactivity demonstrated in our study proposes a novel mechanism by which TNF- $\alpha$ activation might be involved, viaincreased oxidative stress, in the pathophysiology of vascular dysfunction in patients with obesity-related MetS.

The degree to which any particular adipose-derived inflammatory mediator enters the blood stream and plays a role in metabolic and cardiovascular disorders has not yet been established [109].

An emerging area of interest is the role of perivascular adipose tissue (PVAT) in regulating local vascular tone [110]. The presence of adipose tissue around the heart and the great vessels has been recently recognized as an independent cardiovascular risk factor. The perivascular adipose tissue (PVAT) is the potential source of the mediators leading to obesity-related vascular damage.

Because of the absence of the fascial boundaries, epicardial adipose tissue may locally interact and affect the coronary arteries and myocardium through paracrine actions of pro- and anti-inflammatory adipokines and other bioactive molecules [111].

Thus, the epicardial and perivascular adipose tissue could mechanically and functionally modulate the function of the myocardium and vasculature thereby possibly playing a role in obesity-related atherosclerosis [112].

Several studies demonstrated that epicardial fat produces a number of bioactive molecules as well as TNFalpha, IL-6, IL-1, resistin, free fatty acid, and adiponectin that could affect cardiovascular morphology and function [113-117].
Moreover, recent studies have shown that perivascular adipocytes physiologically exert anticontractile effects which are both NO dependent and independent.

In particular, adipokines released from fat depots have local rather than systemic vasoregulatory effects, a mechanism defined as vasocrine signaling. In healthy subjects, PVAT seems to mediate an anticontractile effect in mediumand small-sized arteries [118, 119].

Adiponectin is the main candidate for this role based on the finding that the anticontractile properties of the fat are abolished entirely with an adiponectin type 1 receptor blocking fragment [119].

Importantly, this effect of PVAT is lost in patients with obesity-related metabolic syndrome, and conformational changes in perivascular fat have been shown to associate with lots of its anticontractile properties likely due to inflammatory, vasoconstrictor, and oxidative mechanisms. Recent studies has demonstrated a positive correlation between epicardial adipose tissues volume and coronary atherosclerosis using multislice computed tomography [120].

Moreover, Hosogai and colleagues demonstrated that PVAT of obese mice is hypoxic and that hypoxia stimulated an inflammatory phenotype and a loss of the anticontractile properties. This effect is mediated by endoplasmic reticulum stress and posttranscriptional regulation. Because hypoxia is a prominent feature of adipose tissue in obese individuals and is thought to cause adipocyte dysfunction and tissue inflammation [121], these findings further support a prominent role for PVAT-derived inflammatory cytokines in adversely modulating vascular function.

Of note, the obese phenotype could be reproduced by adding the inflammatory cytokines IL- 6 or TNF- $\alpha$ to the PVAT around healthy vessels, which in turn could be blocked by cytokine antagonists.

In conclusion, these studies suggest that PVAT in obese individuals predominately exerts influence that contributes to vascular disease and may act both locally (paracrine) and downstream (vasocrine), through outside-to-inside signaling.

\section{Renal Effects of Obesity}

The most common types of morphological renal lesions observed in renal biopsies of obese patients are mainly focal and segmental glomerulosclerosis and glomerulomegaly [122]. The podocyte foot process effacement described in the ADP knockoutmice probably represents an earlier stage of kidney involvement. Early changes occurring in the presence of only mild metabolic abnormalities and mild hypertension in the absence of diabetes mellitus include increased glomerular cell proliferation, increased mesangial matrix, thicker basement membrane, and increased expression of glomerular transforming growth factor-beta [50].

In a recent study, Lamacchia and colleagues demonstrated that para- and perirenal fat thickness is an independent predictor of kidney dysfunction and increased renal resistance in patients with type 2 diabetes [123].

The mechanisms of obesity-induced renal injury are not fully understood and are likely to involve a combination of 
hemodynamic and metabolic abnormalities. Many factors contribute to the increase in both glomerular filtration rate (GFR) and rise in renal plasma flow (RPF) observed in obese patients. The increased protein intake determines a rise in GFR [124]. Moreover, IR causes increase in the efferent arteriolar pressure because the reduction of noradrenalineinduced efferent arteriolar constriction by insulin action is blunted. Subsequently, the transcapillary pressure gradient increases resulting in hyperfiltration [124]. Augmented filtration determines microalbuminuria. Moreover, insulin stimulates the synthesis of IGF-1 and IGF-2, both promoting glomerular hypertrophy $[122,124]$. The rapid and parallel increases in the prevalence of end-stage renal disease and obesity in the past two decades suggest that obesity may be a major risk for kidney disease through other mechanisms than diabetes and hypertension (see Figure 1) [125]. Additionally, obesity increases the risk of development of renal diseases from different aetiologies, such as primary immunoglobulin A nephritis or unilateral nephrectomy $[126,127]$. Whether weight loss, in the long term, can slow down the progression of renal damage or reverse it is unknown at the present time. In the short term, weight loss usually leads to reduction of the proteinuria. Antiproteinuric effects of weight loss are also observed in obese patients with nephropathies due to other causes [126].

\section{Mechanisms of Vascular Damage in Obesity}

Abdominal obesity is well recognized as a major risk factor for CVD, but the underlying mechanisms of vascular damage in obesity remain poorly understood.

One of the most important mechanisms by which obesity leads to the development of vascular diseases is the development of IR in skeletal muscle, adipose tissue, and liver. The adipose tissue excess, particularly visceral fat, is associated with a continuous production of mediators that impair insulin action in skeletal muscle, like free fatty acids (FFAs), and is associated with a decreased production of adiponectin, a mediator known to improve insulin sensitivity [128]. Plasminogen activator inhibitor-1 (PAI-1) is typically increased in the obesity/IR state and plays an important role in the genesis of vascular abnormalities [129]. In addition, obesity and IR are frequently associated with altered coagulation/fibrinolysis. In combination, all of the above abnormalities create a state of constant and progressive damage to the vascular wall, manifested by a low-grade progressive inflammatory process [130]. Endothelial dysfunction is defined as paradoxical or inadequate endothelialmediated vasodilation and is characterized by loss of balance between vasoconstrictors and vasodilators, increased oxidative stress, and elevated expression of proinflammatory and prothrombotic factors.

Endothelial dysfunction is a fundamental initial step in the development and progression of atherosclerosis and has been considered an important event in the development of microvascular complications [130].

Adipose tissue can produce a significant amount of compounds able to affect endothelial function; the ability of adipokines to directly affect vascular homeostasis may

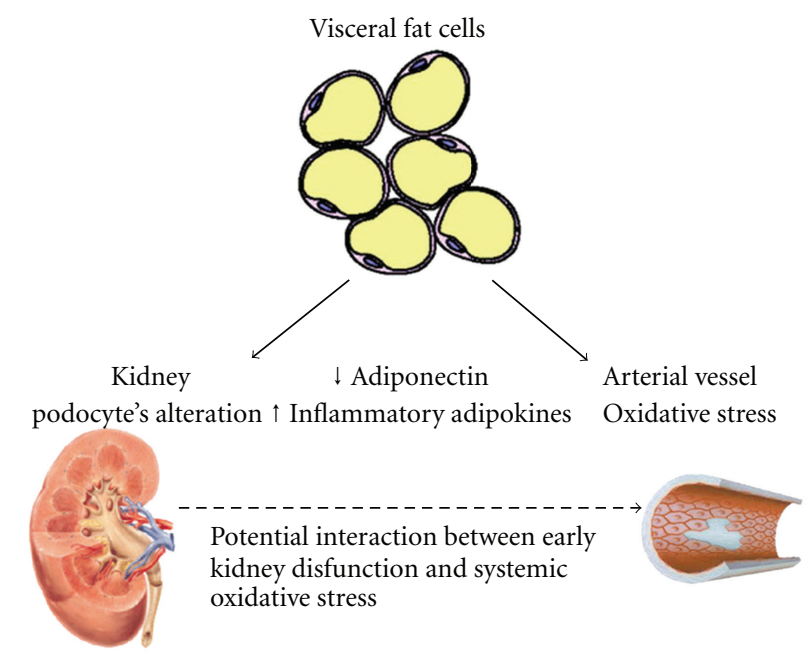

Figure 1: Possible dynamic interactions between obesity, CKD, and CVD. The increase of visceral mass determines both decreased production of $\mathrm{ADN}$ and increased production of inflammatory adipokines which result in increased insulin resistance.

represent an important mechanistic basis of vascular disease in patients with obesity. Central obesity is associated with increased levels of free fatty acid (FFA) that induces endothelial dysfunction by effect of insulin-mediated vasodilatation in humans and by impairment of nitric oxide-independent mechanism mediated by reduction of potassium-mediated vasodilatation [131]. The increased levels of FFA from the more lipolytically active intra-abdominal adipocytes decreased insulin sensitivity through the intracellular insulin signaling [132].

Moreover, FFAs are capable of utilizing the innate immune receptor TLR4 to induce proinflammatory cytokine expression in macrophages and adipocytes. In particular, the TLR4 signaling appears to be required for a component of insulin resistance induced by FFAs in adipocytes and in vivo after lipid infusion and high-fat diets [133].

Moreover, TNF alpha overproduction in obesity mediates the increased endothelial permeability by activating NADPH oxidase [129] and also inhibits transcriptional, as well as posttranscriptional, eNOS gene expression an effect that can account for the endothelial dysfunction.

In obese patients, an increase in reactive oxygen species has been demonstrated and could be the link between the low-grade inflammation, platelet activation, and nitric oxide destruction.

In IR and obesity, the dyslipidaemia is characterized by a different composition and distribution of LDL cholesterol, resulting in an increased concentration of the more atherogenic small dense LDL. Small dense LDL particles can move through endothelial fenestrations, entering the subendothelial space where inflammation and transformation into plaque can occur [134]. Moreover, the ox-LDL is mostly taken up by macrophage scavenger receptors, rather than the normal LDL receptor pathway, thus inducing atherosclerosis. 


\section{Vascular Dysfunction in Insulin Resistance and Metabolic Syndrome}

Hyperinsulinemia and IR are established metabolic features of obesity. Vasodilator actions of insulin to stimulate production of nitric oxide (NO) from vascular endothelium lead to increased blood flow that further enhances glucose uptake in skeletal muscle $[135,136]$. Insulin binding to its cognate receptor activates two major branches of insulin signal transduction network. Metabolic actions of insulin tend to be mediated by phosphatidylinositol 3-kinase- (PI3K-) dependent signaling pathways, whereas the nonmetabolic insulin signalling pathways (MAPK-dependent insulin signaling pathways) typically regulate growth, mitogenesis, and differentiation $[137,138]$ and also regulate the secretion of the vasoconstrictor endothelin-1 (ET-1) from endothelium.

The PI3K-dependent metabolic actions of insulin directly promote glucose uptake in skeletal muscle by stimulating translocation of insulin responsive glucose transporters (GLUT4). At the same time, PI3K-dependent vascular actions of insulin to increase blood flow and capillary recruitment substantially contribute to promoting glucose disposal under healthy conditions [3].

ET-1, a potent vasoconstrictor synthesized and secreted by vascular endothelium, plays an important role in endothelial dysfunction.

In humans, insulin-stimulated ET-1 production may influence skeletal muscle glucose disposal, and ET-1 infusion induces peripheral insulin resistance. NO, however, is known to inhibit ET-1 production and action [139]. Consequently, under healthy conditions, the effects of insulin-stimulated ET-1 on the metabolic actions of insulin are likely to be offset by insulin-stimulated production of NO [140].

IR is characterized by selective impairment in PI3Kdependent signalling in both metabolic and vascular insulin target tissues, and the diminished sensitivity to the actions of insulin in vascular endothelium contributes importantly to the clinical phenotype of this condition [3].

In insulin-resistant states, the insulin-mediated ET-1 secretion is augmented, and blockade of ET-1 receptors significantly improves insulin sensitivity and peripheral glucose uptake in the context of IR $[141,142]$.

Another important mechanism of insulin action is the increased microvascular perfusion of skeletal muscle leading to increased muscle blood flow and glucose uptake.

While the majority of previous studies have focused on the endothelium-dependent effect of insulin, we have suggested that the blunted vasodilator responsiveness to the exogenous NO seen in obese patients during hyperinsulinemia could be related to the facilitatory action physiologically exerted by insulin on vasorelaxation within VSMCs. Indeed, insulin enhances vasodilator responsiveness by reducing $\mathrm{Ca}^{2+}$ concentration in VSMCs, via activation of the $\mathrm{Na}^{+}, \mathrm{K}^{+}-$ ATPase pump [143]. Similarly, in human VSMCs, insulin inactivates the small GTPase RhoA and its target, Rhokinase, thereby leading to decreased phosphorylation of myosin light chain and subsequent vasodilation [144].

In a recent study, we have demonstrated that insulin exerts a facilitatory effect on vascular reactivity to a variety of vasoactive molecules (acetylcholine, sodium nitroprusside, and verapamil) by the increased delivery of substrates in skeletal muscle of healthy subjects, but in contrast with the results obtained in healthy subjects, the responsiveness of these vasodilators was not enhanced during hyperinsulinemia in the group of patients with the metabolic syndrome due to increased oxidative stress [145].

\section{Conclusion}

The rapid growth of obesity is contributing to an "epidemic" in Mets and diabetes and related renal and cardiovascular complications. Adipose tissue is an endocrine organ which produces a variety of "adipokines." Clinical management should be aimed to reduce risk factors by encouraging lifestyle modifications such as diet, aerobic exercise, and healthy eating as well as pharmacologic intervention to improve insulin sensitivity, dyslipidemia, and blood pressure control and protect the kidney from further injury. At the present time, there are few drugs available to produce significant long-term weight loss. Weight loss of about $10 \%$ can improve insulin sensitivity and lower blood pressure, reduce serum lipid levels, decrease plasma inflammatory cytokines, and increase circulating AND [97, 146]. Finally, an emerging area of interest is the potential role of the podocytespecific drugs. Further research on adipokines action on renal cells is needed and will lead to novel treatment strategies aimed to fight the increased incidence of obesityrelated chronic kidney and cardiovascular diseases.

\section{Conflict of Interest}

The authors declare no conflict of interest.

\section{References}

[1] T. L. S. Visscher and J. C. Seidell, "The public health impact of obesity," Annual Review of Public Health, vol. 22, pp. 355375, 2001.

[2] S. A. French, M. Story, and R. W. Jeffery, "Environmental influences on eating and physical activity," Annual Review of Public Health, vol. 22, pp. 309-335, 2001.

[3] R. Muniyappa, M. Montagnani, K. K. Koh, and M. J. Quon, "Cardiovascular actions of insulin," Endocrine Reviews, vol. 28, no. 5, pp. 463-491, 2007.

[4] A. Avogaro and S. V. De Kreutzenberg, "Mechanisms of endothelial dysfunction in obesity," Clinica Chimica Acta, vol. 360, no. 1-2, pp. 9-26, 2005.

[5] J. R. Henegar, S. A. Bigler, L. K. Henegar, S. C. Tuagi, and J. E. Hall, "Functional and structural changes in the kidney in the early stages of obesity," Journal of the American Society of Nephrology, vol. 12, no. 6, pp. 1211-1217, 2001.

[6] A. S. Go, G. M. Chertow, D. Fan, C. E. McCulloch, and C. Y. Hsu, "Chronic kidney disease and the risks of death, cardiovascular events, and hospitalization," New England Journal of Medicine, vol. 351, no. 13, pp. 1296-1370, 2004.

[7] M. G. Shlipak, M. J. Sarnak, R. Katz et al., "Cystatin C and the risk of death and cardiovascular events among elderly persons," New England Journal of Medicine, vol. 352, no. 20, pp. 2049-2060, 2005. 
[8] J. Danziger, "Importance of low-grade albuminuria," Mayo Clinic Proceedings, vol. 83, no. 7, pp. 806-812, 2008.

[9] D. De Zeeuw, H. H. Parving, and R. H. Henning, "Microalbuminuria as an early marker for cardiovascular disease," Journal of the American Society of Nephrology, vol. 17, no. 8, pp. 2100-2105, 2006.

[10] C. Y. Hsu, C. E. McCulloch, C. Iribarren, J. Darbinian, and A. S. Go, "Body mass index and risk for end-stage renal disease," Annals of Internal Medicine, vol. 144, no. 1, pp. 21-28, 2006.

[11] R. N. Foley, P. S. Parfrey, and M. J. Sarnak, "Clinical epidemiology of cardiovascular disease in chronic renal disease," American Journal of Kidney Diseases, vol. 32, no. 5, pp. S112-S119, 1998.

[12] A. Lindner, B. Charra, D. J. Sherrard, and B. H. Scribner, "Accelerated atherosclerosis in prolonged maintenance hemodialysis," New England Journal of Medicine, vol. 290, no. 13, pp. 697-701, 1974.

[13] R. Vanholder, Z. Massy, A. Argiles et al., "Chronic kidney disease as cause of cardiovascular morbidity and mortality," Nephrology Dialysis Transplantation, vol. 20, pp. 1048-1056, 2005.

[14] R. M. A. Henry, P. J. Kostense, G. Bos et al., "Mild renal insufficiency is associated with increased cardiovascular mortality," Kidney International, vol. 62, no. 4, pp. 1402 1407, 2002.

[15] T. Ninomiya, Y. Kiyohara, M. Kubo et al., "Metabolic syndrome and CKD in a general Japanese population: The Hisayama Study," American Journal of Kidney Diseases, vol. 48, no. 3, pp. 383-391, 2006.

[16] J. Chen, P. Muntner, L. L. Hamm et al., "The metabolic syndrome and chronic kidney disease in U.S. adults," Annals of Internal Medicine, vol. 140, no. 3, pp. 167-174, 2004.

[17] N. Perico, I. Codreanu, A. Schieppati, and G. Remuzzi, "Prevention of progression and remission/regression strategies for chronic renal diseases: can we do better now than five years ago?" Kidney International, vol. 68, no. 98, supplement, pp. S21-S24, 2005.

[18] H. Bahrami, D. A. Bluemke, R. Kronmal et al., "Novel metabolic risk factors for incident heart failure and their relationship with obesity. The MESA (Multi-Ethnic Study of Atherosclerosis) Study," Journal of the American College of Cardiology, vol. 51, no. 18, pp. 1775-1783, 2008.

[19] K. Wachtell, H. Ibsen, M. H. Olsen et al., "Albuminuria and cardiovascular risk in hypertensive patients with left ventricular hypertrophy: the LIFE study," Annals of Internal Medicine, vol. 139, no. 11, pp. 901-906, 2003.

[20] K. E. Wellen and G. S. Hotamisligil, "Obesity-induced inflammatory changes in adipose tissue," Journal of Clinical Investigation, vol. 112, no. 12, pp. 1785-1788, 2003.

[21] L. K. M. Summers, J. S. Samra, and K. N. Frayn, "Impaired postprandial tissue regulation of blood flow in insulin resistance: a determinant of cardiovascular risk?" Atherosclerosis, vol. 147, no. 1, pp. 11-15, 1999.

[22] N. Ouchi, S. Kihara, Y. Arita et al., "Novel modulator for endothelial adhesion molecules: adipocyte-derived plasma protein adiponectin," Circulation, vol. 100, no. 25, pp. 24732476, 1999.

[23] Y. Arita, S. Kihara, N. Ouchi et al., "Paradoxical decrease of an adipose-specific protein, adiponectin, in obesity," Biochemical and Biophysical Research Communications, vol. 257, no. 1, pp. 79-83, 1999.

[24] K. Hotta, T. Funahashi, Y. Arita et al., "Plasma concentrations of a novel, adipose-specific protein, adiponectin, in type 2 diabetic patients," Arteriosclerosis, Thrombosis, and Vascular Biology, vol. 20, no. 6, pp. 1595-1599, 2000.

[25] E. E. Kershaw and J. S. Flier, "Adipose tissue as an endocrine organ," Journal of Clinical Endocrinology and Metabolism, vol. 89, no. 6, pp. 2548-2556, 2004.

[26] A. R. Nawrocki and P. E. Scherer, "The delicate balance between fat and muscle: adipokines in metabolic disease and musculoskeletal inflammation," Current Opinion in Pharmacology, vol. 4, no. 3, pp. 281-289, 2004.

[27] P. J. Havel, "Update on adipocyte hormones: regulation of energy balance and carbohydrate/lipid metabolism," Diabetes, vol. 53, no. 1, pp. S143-S151, 2004.

[28] K. Shimada, T. Miyazaki, and H. Daida, "Adiponectin and atherosclerotic disease," Clinica Chimica Acta, vol. 344, no. 1-2, pp. 1-12, 2004.

[29] B. Becker, F. Kronenberg, J. T. Kielstein et al., "Renal insulin resistance syndrome, adiponectin and cardiovascular events in patients with kidney disease: the mild and moderate kidney disease study," Journal of the American Society of Nephrology, vol. 16, no. 4, pp. 1091-1098, 2005.

[30] C. Tsioufis, K. Dimitriadis, D. Chatzis et al., "Relation of microalbuminuria to adiponectin and augmented C-reactive protein levels in men with essential hypertension," American Journal of Cardiology, vol. 96, no. 7, pp. 946-951, 2005.

[31] Y. Yano, S. Hoshide, J. Ishikawa et al., "Differential impacts of adiponectin on low-grade albuminuria between obese and nonobese persons without diabetes," Journal of Clinical Hypertension, vol. 9, no. 10, pp. 775-782, 2007.

[32] K. Sharma, S. RamachandraRao, G. Qiu et al., "Adiponectin regulates albuminuria and podocyte function in mice," Journal of Clinical Investigation, vol. 118, no. 5, pp. 16451656, 2008.

[33] J. Koshimura, H. Fujita, T. Narita et al., "Urinary adiponectin excretion is increased in patients with overt diabetic nephropathy," Biochemical and Biophysical Research Communications, vol. 316, no. 1, pp. 165-169, 2004.

[34] A. Jorsal, L. Tarnow, J. Frystyk et al., "Serum adiponectin predicts all-cause mortality and end stage renal disease in patients with type I diabetes and diabetic nephropathy," Kidney International, vol. 74, no. 5, pp. 649-654, 2008.

[35] K. Sharma, "The link between obesity and albuminuria: adiponectin and podocyte dysfunction," Kidney International, vol. 76, no. 2, pp. 145-148, 2009.

[36] V. Eremina, H. J. Baelde, and S. E. Quaggin, "Role of the VEGF-a signaling pathway in the glomerulus: evidence for crosstalk between components of the glomerular filtration barrier," Nephron Physiology, vol. 106, no. 2, pp. p32-p37, 2007.

[37] V. Eremina, J. A. Jefferson, J. Kowalewska et al., "VEGF inhibition and renal thrombotic microangiopathy," New England Journal of Medicine, vol. 358, no. 11, pp. 1129-1136, 2008.

[38] J. M. Friedman and J. L. Halaas, "Leptin and the regulation of body weight in mammals," Nature, vol. 395, no. 6704, pp. 763-770, 1998.

[39] J. Ren, "Leptin and hyperleptinemia-from friend to foe for cardiovascular function," Journal of Endocrinology, vol. 181, no. 1, pp. 1-10, 2004.

[40] S. Söderberg, B. Ahrén, J. H. Jansson et al., "Leptin is associated with increased risk of myocardial infarction," Journal of Internal Medicine, vol. 246, no. 4, pp. 409-418, 1999.

[41] A. Corsonello, F. Perticone, A. Malara et al., "Leptindependent platelet aggregation in healthy, overweight and 
obese subjects," International Journal of Obesity, vol. 27, no. 5, pp. 566-573, 2003.

[42] S. Konstantinides, K. Schäfer, S. Koschnick, and D. J. Loskutoff, "Leptin-dependent platelet aggregation and arterial thrombosis suggests a mechanism for atherothrombotic disease in obesity," Journal of Clinical Investigation, vol. 108, no. 10, pp. 1533-1540, 2001.

[43] S. Konstantinides, K. Schäfer, and D. J. Loskutoff, "The prothrombotic effects of leptin: possible implications for the risk of cardiovascular disease in obesity," Annals of the New York Academy of Sciences, vol. 947, pp. 134-142, 2001.

[44] S. Loffreda, S. Q. Yang, H. Z. Lin et al., "Leptin regulates proinflammatory immune responses," FASEB Journal, vol. 12, no. 1, pp. 57-65, 1998.

[45] F. Parhami, Y. Tintut, A. Ballard, A. M. Fogelman, and L. L. Demer, "Leptin enhances the calcification of vascular cells artery wall as a target of leptin," Circulation Research, vol. 88, no. 9, pp. 954-960, 2001.

[46] M. Laimer, C. F. Ebenbichler, S. Kaser et al., "Markers of chronic inflammation and obesity: a prospective study on the reversibility of this association in middle-aged women undergoing weight loss by surgical intervention," International Journal of Obesity, vol. 26, no. 5, pp. 659-662, 2002.

[47] M. Maachi, L. Piéroni, E. Bruckert et al., "Systemic low-grade inflammation is related to both circulating and adipose tissue TNF $\alpha$, leptin and IL-6 levels in obese women," International Journal of Obesity, vol. 28, no. 8, pp. 993-997, 2004.

[48] L. U. Monzillo, O. Hamdy, E. S. Horton et al., "Effect of lifestyle modification on adipokine levels in obese subjects with insulin resistance," Obesity Research, vol. 11, no. 9, pp. 1048-1054, 2003.

[49] D. K. Papafragkaki and G. Tolis, "Obesity and renal disease: a possible role of leptin,” Hormones, vol. 4, no. 2, pp. 90-95, 2005.

[50] J. R. Henegar, S. A. Bigler, L. K. Henegar, S. C. Tyagi, and J. E. Hall, "Functional and structural changes in the kidney in the early stages of obesity," Journal of the American Society of Nephrology, vol. 12, no. 6, pp. 1211-1217, 2001.

[51] N. Kambham, G. S. Markowitz, A. M. Valeri, J. Lin, and V. D. D’Agati, "Obesity-related glomerulopathy: an emerging epidemic," Kidney International, vol. 59, no. 4, pp. 14981509, 2001.

[52] P. Stenvinkel, "Leptin and its clinical implications in chronic renal failure," Mineral and Electrolyte Metabolism, vol. 25, no. 4-6, pp. 298-302, 1999.

[53] R. D. Adelman, "Obesity and renal disease," Current Opinion in Nephrology and Hypertension, vol. 11, no. 3, pp. 331-335, 2002.

[54] M. Kojima, H. Hosoda, Y. Date, M. Nakazato, H. Matsuo, and K. Kangawa, "Ghrelin is a growth-hormone-releasing acylated peptide from stomach," Nature, vol. 402, no. 6762, pp. 656-660, 1999.

[55] K. Takaya, H. Ariyasu, N. Kanamoto et al., "Ghrelin strongly stimulates growth hormone (GH) release in humans," Journal of Clinical Endocrinology and Metabolism, vol. 85, no. 12, pp. 4908-4911, 2000.

[56] M. Iantorno, H. Chen, J. A. Kim et al., "Ghrelin has novel vascular actions that mimic PI 3-kinase-dependent actions of insulin to stimulate production of NO from endothelial cells," American Journal of Physiology, vol. 292, no. 3, pp. E756-E764, 2007.

[57] M. Tesauro, M. Iantorno, F. Schinzari, and C. Cardillo, "Vascular effects of insulin and their relation to endothelial dysfunction, insulin resistance and hypertension," Current Hypertension Reviews, vol. 5, no. 4, pp. 251-261, 2009.

[58] M. Tesauro, F. Schinzari, M. Iantorno et al., "Ghrelin improves endothelial function in patients with metabolic syndrome," Circulation, vol. 112, no. 19, pp. 2986-2992, 2005.

[59] P. Cano, D. P. Cardinali, M. J. Ríos-Lugo, M. P. FernándezMateos, C. F. Reyes Toso, and A. I. Esquifino, "Effect of a high-fat diet on 24-hour pattern of circulating adipocytokines in rats," Obesity, vol. 17, no. 10, pp. 1866-1871, 2009.

[60] A. H. Berg, T. P. Combs, X. Du, M. Brownlee, and P. E. Scherer, "The adipocyte-secreted protein Acrp30 enhances hepatic insulin action," Nature Medicine, vol. 7, no. 8, pp. 947-953, 2001.

[61] J. Fruebis, T. S. Tsao, S. Javorschi et al., "Proteolytic cleavage product of $30-\mathrm{kDa}$ adipocyte complement-related protein increases fatty acid oxidation in muscle and causes weight loss in mice," Proceedings of the National Academy of Sciences of the United States of America, vol. 98, no. 4, pp. 2005-2010, 2001.

[62] G. S. Hotamisligil, N. S. Shargill, and B. M. Spiegelman, "Adipose expression of tumor necrosis factor- $\alpha$ : direct role in obesity-linked insulin resistance," Science, vol. 259, no. 5091, pp. 87-91, 1993.

[63] J. R. Sowers, "Endocrine functions of adipose tissue: focus on adiponectin," Clinical Cornerstone, vol. 9, no. 1, pp. 32-38, 2008.

[64] G. H. Goossens, E. E. Blaak, and M. A. Van Baak, "Possible involvement of the adipose tissue renin-angiotensin system in the pathophysiology of obesity and obesity-related disorders," Obesity Reviews, vol. 4, no. 1, pp. 43-55, 2003.

[65] K. L. Comstock, K. A. Krown, M. T. Page et al., "LPS-induced TNF- $\alpha$ release from and apoptosis in rat cardiomyocytes: obligatory role for CD14 in mediating the LPS response," Journal of Molecular and Cellular Cardiology, vol. 30, no. 12, pp. 2761-2775, 1998.

[66] D. L. Mann, "Stress-activated cytokines and the heart: from adaptation to maladaptation," Annual Review of Physiology, vol. 65, pp. 81-101, 2003.

[67] G. Torre-Amione, S. Kapadia, J. Lee et al., "Tumor necrosis factor- $\alpha$ and tumor necrosis factor receptors in the failing human heart," Circulation, vol. 93, no. 4, pp. 704-711, 1996.

[68] T. Yokoyama, L. Vaca, R. D. Rossen, W. Durante, P. Hazarika, and D. L. Mann, "Cellular basis for the negative inotropic effects of tumor necrosis factor- $\alpha$ in the adult mammalian heart," Journal of Clinical Investigation, vol. 92, no. 5, pp. 2303-2312, 1993.

[69] H. H. Sigusch, M. H. Lehmann, U. Schnittler, D. Reinhardt, and H. R. Figulla, "Tumour necrosis factor- $\alpha$ expression in idiopathic dilated cardiomyopathy: correlation to myocardial inflammatory activity," Cytokine, vol. 12, no. 8, pp. 12611266, 2000.

[70] S. Kapadia, J. Lee, G. Torre-Amione, H. H. Birdsall, T. S. Ma, and D. L. Mann, "Tumor necrosis factor- $\alpha$ gene and protein expression in adult feline myocardium after endotoxin administration," Journal of Clinical Investigation, vol. 96, no. 2, pp. 1042-1052, 1995.

[71] T. Kubota, C. F. McTiernan, C. S. Frye, A. J. Demetris, and A. M. Feldman, "Cardiac-specific overexpression of tumor necrosis factor-alpha causes lethal myocarditis in transgenic mice," Journal of Cardiac Failure, vol. 3, no. 2, pp. 117-124, 1997.

[72] M. R. Bristow, "Tumor necrosis factor- $\alpha$ and cardiomyopathy," Circulation, vol. 97, no. 14, pp. 1340-1341, 1998. 
[73] R. Shibata, N. Ouchi, M. Ito et al., "Adiponectin-mediated modulation of hypertrophic signals in the heart," Nature Medicine, vol. 10, no. 12, pp. 1384-1389, 2004.

[74] F. Dong and J. Ren, "Adiponectin improves cardiomyocyte contractile fnction in $\mathrm{db} / \mathrm{db}$ diabetic obese mice," Obesity, vol. 17, no. 2, pp. 262-268, 2009.

[75] N. Ouchi, S. Kihara, T. Funahashi, Y. Matsuzawa, and K. Walsh, "Obesity, adiponectin and vascular inflammatory disease," Current Opinion in Lipidology, vol. 14, no. 6, pp. 561-566, 2003.

[76] H. Chen, M. Montagnani, T. Funahashi, I. Shimomura, and M. J. Quon, "Adiponectin stimulates production of nitric oxide in vascular endothelial cells," Journal of Biological Chemistry, vol. 278, no. 45, pp. 45021-45026, 2003.

[77] K. K. Y. Cheng, K. S. L. Lam, Y. Wang et al., "Adiponectininduced endothelial nitric oxide synthase activation and nitric oxide production are mediated by APPL1 in endothelial cells," Diabetes, vol. 56, no. 5, pp. 1387-1394, 2007.

[78] S. J. Hong, C. G. Park, H. S. Seo, D. J. Oh, and Y. M. Ro, "Associations among plasma adiponectin, hypertension, left ventricular diastolic function and left ventricular mass index," Blood Pressure, vol. 13, no. 4, pp. 236-242, 2004.

[79] P. C. Schulze, J. Kratzsch, A. Linke et al., "Elevated serum levels of leptin and soluble leptin receptor in patients with advanced chronic heart failure," European Journal of Heart Failure, vol. 5, no. 1, pp. 33-40, 2003.

[80] S. Söderberg, B. Ahrén, J. H. Jansson et al., "Leptin is associated with increased risk of myocardial infarction," Journal of Internal Medicine, vol. 246, no. 4, pp. 409-418, 1999.

[81] A. M. Wallace, A. D. McMahon, C. J. Packard et al., "Plasma leptin and the risk of cardiovascular disease in the West of Scotland Coronary Prevention Study (WOSCOPS)," Circulation, vol. 104, no. 25, pp. 3052-3056, 2001.

[82] D. E. Flanagan, J. C. Vaile, G. W. Petley et al., "Gender differences in the relationship between leptin, insulin resistance and the autonomic nervous system," Regulatory Peptides, vol. 140, no. 1-2, pp. 37-42, 2007.

[83] C. Menendez, R. Baldelli, M. Lage et al., "The in vitro secretion of human leptin is gender-dependent but independent of the body mass index of the donors," European Journal of Endocrinology, vol. 143, no. 5, pp. 711-714, 2000.

[84] P. F. Bodary, R. J. Westrick, K. J. Wickenheiser, Y. Shen, and D. T. Eitzman, "Effect of leptin on arterial thrombosis following vascular injury in mice," Journal of the American Medical Association, vol. 287, no. 13, pp. 1706-1709, 2002.

[85] J. P. Cooke and R. K. Oka, "Does leptin cause vascular disease?” Circulation, vol. 106, no. 15, pp. 1904-1905, 2002.

[86] G. N. Chaldakov, M. Fiore, I. S. Stankulov et al., "NGF, BDNF, leptin, and mast cells in human coronary atherosclerosis and metabolic syndrome," Archives of Physiology and Biochemistry, vol. 109, no. 4, pp. 357-360, 2001.

[87] N.-F. Chu, D. Spiegelman, N. Rifai, G. S. Hotamisligil, and E. B. Rimm, "Glycemic status and soluble tumor necrosis factor receptor levels in relation to plasma leptin concentrations among normal weight and overweight US men," International Journal of Obesity, vol. 24, no. 9, pp. 1085-1092, 2000.

[88] M. R. Sierra-Honigmann, A. K. Nath, C. Murakami et al., "Biological action of leptin as an angiogenic factor," Science, vol. 281, no. 5383, pp. 1683-1686, 1998.

[89] A. Singhal, S. Farooqi, T. J. Cole et al., "Influence of leptin on arterial distensibility: a novel link between obesity and cardiovascular disease?" Circulation, vol. 106, no. 15, pp. 1919-1924, 2002.

[90] N. Nagaya, M. Uematsu, M. Kojima et al., "Elevated circulating level of ghrelin in cachexia associated with chronic heart failure: relationships between ghrelin and anabolic/catabolic factors," Circulation, vol. 104, no. 17, pp. 2034-2038, 2001.

[91] S. Frascarelli, S. Ghelardoni, S. Ronca-Testoni, and R. Zucchi, "Effect of ghrelin and synthetic growth hormone secretagogues in normal and ischemic rat heart," Basic Research in Cardiology, vol. 98, no. 6, pp. 401-405, 2003.

[92] N. Nagaya, M. Uematsu, M. Kojima et al., "Chronic administration of ghrelin improves left ventricular dysfunction and attenuates development of cardiac cachexia in rats with heart failure," Circulation, vol. 104, no. 12, pp. 1430-1435, 2001.

[93] N. Nagaya, M. Kojima, M. Uematsu et al., "Hemodynamic and hormonal effects of human ghrelin in healthy volunteers," American Journal of Physiology - Regulatory Integrative and Comparative Physiology, vol. 280, no. 5, pp. R1483R1487, 2001.

[94] N. Nagaya and K. Kangawa, "Therapeutic potential of Ghrelin in the treatment of heart failure," Drugs, vol. 66, no. 4, pp. 439-448, 2006.

[95] N. Nagaya, K. Miyatake, M. Uematsu et al., "Hemodynamic, renal, and hormonal effects of ghrelin infusion in patients with chronic heart failure," Journal of Clinical Endocrinology and Metabolism, vol. 86, no. 12, pp. 5854-5859, 2001.

[96] N. Nagaya, J. Moriya, Y. Yasumura et al., "Effects of ghrelin administration on left ventricular function, exercise capacity, and muscle wasting in patients with chronic heart failure," Circulation, vol. 110, no. 24, pp. 3674-3679, 2004.

[97] N. Kamei, K. Tobe, R. Suzuki et al., "Overexpression of monocyte chemoattractant protein-1 in adipose tissues causes macrophage recruitment and insulin resistance," Journal of Biological Chemistry, vol. 281, no. 36, pp. 2660226614, 2006.

[98] H. Xu, G. T. Barnes, Q. Yang et al., "Chronic inflammation in fat plays a crucial role in the development of obesity-related insulin resistance," Journal of Clinical Investigation, vol. 112, no. 12, pp. 1821-1830, 2003.

[99] S. P. Weisberg, D. McCann, M. Desai, M. Rosenbaum, R. L. Leibel, and A. W. Ferrante, "Obesity is associated with macrophage accumulation in adipose tissue," Journal of Clinical Investigation, vol. 112, no. 12, pp. 1796-1808, 2003.

[100] J. R. Sowers, "Metabolic risk factors and renal disease," Kidney International, vol. 71, no. 8, pp. 719-720, 2007.

[101] S. P. Weisberg, D. McCann, M. Desai, M. Rosenbaum, R. L. Leibel, and A. W. Ferrante Jr., "Obesity is associated with macrophage accumulation in adipose tissue," Journal of Clinical Investigation, vol. 112, no. 12, pp. 1796-1808, 2003.

[102] C. A. Curat, A. Miranville, C. Sengenès et al., "From blood monocytes to adipose tissue-resident macrophages: induction of diapedesis by human mature adipocytes," Diabetes, vol. 53, no. 5, pp. 1285-1292, 2004.

[103] R. Cancello, J. Tordjman, C. Poitou et al., "Increased infiltration of macrophages in omental adipose tissue is associated with marked hepatic lesions in morbid human obesity," Diabetes, vol. 55, no. 6, pp. 1554-1561, 2006.

[104] G. Zeng, F. H. Nystrom, L. V. Ravichandran et al., "Roles for insulin receptor, PI3-kinase, and Akt in insulin-signaling pathways related to production of nitric oxide in human vascular endothelial cells," Circulation, vol. 101, no. 13, pp. 1539-1545, 2000. 
[105] N. Begum and L. Ragolia, "Effect of tumor necrosis factor$\alpha$ on insulin action in cultured rat skeletal muscle cells," Endocrinology, vol. 137, no. 6, pp. 2441-2446, 1996.

[106] T. Suganami, J. Nishida, and Y. Ogawa, "A paracrine loop between adipocytes and macrophages aggravates inflammatory changes: role of free fatty acids and tumor necrosis factor $\alpha$," Arteriosclerosis, Thrombosis, and Vascular Biology, vol. 25, no. 10, pp. 2062-2068, 2005.

[107] G. S. Hotamisligil, "Inflammation and metabolic disorders," Nature, vol. 444, no. 7121, pp. 860-867, 2006.

[108] M. Tesauro, F. Schinzari, V. Rovella et al., "TNF-alpha antagonism improves insulin-stimulated vasodilation in metabolic syndrome," Diabetes Care, vol. 31, no. 7, pp. 1439-1441, 2008.

[109] R. E. Gimeno and L. D. Klaman, "Adipose tissue as an active endocrine organ: recent advances," Current Opinion in Pharmacology, vol. 5, no. 2, pp. 122-128, 2005.

[110] M. Feuerer, L. Herrero, D. Cipolletta et al., "Lean, but not obese, fat is enriched for a unique population of regulatory $\mathrm{T}$ cells that affect metabolic parameters," Nature Medicine, vol. 15, no. 8, pp. 930-939, 2009.

[111] G. Iacobellis, C. R. di Gioia, D. Cotesta et al., "Epicardial adipose tissue adiponectin expression is related to intracoronary adiponectin levels," Hormone and Metabolic Research, vol. 41, no. 3, pp. 227-231, 2009.

[112] G. Barbaro and G. Iacobellis, "Metabolic syndrome associated with HIV and highly active antiretroviral therapy," Current Diabetes Reports, vol. 9, no. 1, pp. 37-42, 2009.

[113] G. Iacobellis, D. Pistilli, M. Gucciardo et al., "Adiponectin expression in human epicardial adipose tissue in vivo is lower in patients with coronary artery disease," Cytokine, vol. 29, no. 6, pp. 251-255, 2005.

[114] T. Mazurek, L. Zhang, A. Zalewski et al., "Human epicardial adipose tissue is a source of inflammatory mediators," Circulation, vol. 108, no. 20, pp. 2460-2466, 2003.

[115] A. R. Baker, N. F. da Silva, D. W. Quinn et al., "Human epicardial adipose tissue expresses a pathogenic profile of adipocytokines in patients with cardiovascular disease," Cardiovascular Diabetology, vol. 5, article 1, 2006.

[116] G. N. Chaldakov, M. Fiore, I. S. Stankulov et al., "Neurotrophin presence in human coronary atherosclerosis and metabolic syndrome: a role for NGF and BDNF in cardiovascular disease?" Progress in Brain Research, vol. 146, pp. 279289, 2004.

[117] J. Kremen, M. Dolinkova, J. Krajickova et al., "Increased subcutaneous and epicardial adipose tissue production of proinflammatory cytokines in cardiac surgery patients: possible role in postoperative insulin resistance," Journal of Clinical Endocrinology and Metabolism, vol. 91, no. 11, pp. 4620-4627, 2006.

[118] Y. J. Gao, Z. H. Zeng, K. Teoh et al., "Perivascular adipose tissue modulates vascular function in the human internal thoracic artery," Journal of Thoracic and Cardiovascular Surgery, vol. 130, no. 4, pp. 1130-1136, 2005.

[119] A. S. Greenstein, K. Khavandi, S. B. Withers et al., "Local inflammation and hypoxia abolish the protective anticontractile properties of perivascular fat in obese patients," Circulation, vol. 119, no. 12, pp. 1661-1670, 2009.

[120] R. Djaberi, J. D. Schuijf, J. M. van Werkhoven, G. Nucifora, J. W. Jukema, and J. J. Bax, "Relation of epicardial adipose tissue to coronary atherosclerosis," American Journal of Cardiology, vol. 102, no. 12, pp. 1602-1607, 2008.
[121] N. Hosogai, A. Fukuhara, K. Oshima et al., "Adipose tissue hypoxia in obesity and its impact on adipocytokine dysregulation," Diabetes, vol. 56, no. 4, pp. 901-911, 2007.

[122] N. Kambham, G. S. Markowitz, A. M. Valeri, J. Lin, and V. D. D'Agati, "Obesity-related glomerulopathy: an emerging epidemic,” Kidney International, vol. 59, no. 4, pp. 14981509, 2001.

[123] O. Lamacchia, V. Nicastro, D. Camarchio et al., "Para and perirenal fat thickness is an independent predictor of chronic kidney disease, increased renal resistance index and hyperuricaemia in type-2 diabetic patients," Nephrology, Dialysis, Transplantation. In press.

[124] D. K. Papafragkaki and G. Tolis, "Obesity and renal disease: a possible role of leptin," Hormones, vol. 4, no. 2, pp. 90-95, 2005.

[125] J. E. Hall, D. W. Jones, J. J. Kuo, A. da Silva, L. S. Tallam, and J. Liu, "Impact of the obesity epidemic on hypertension and renal disease," Current Hypertension Reports, vol. 5, no. 5, pp. 386-392, 2003.

[126] E. Morales, M. A. Valero, M. León, E. Hernández, and M. Praga, "Beneficial effects of weight loss in overweight patients with chronic proteinuric nephropathies," American Journal of Kidney Diseases, vol. 41, no. 2, pp. 319-327, 2003.

[127] M. Praga, E. Hernandez, J. C. Herrero et al., "Influence of obesity on the appearance of proteinuria and renal insufficiency after unilateral nephrectomy," Kidney International, vol. 58, no. 5, pp. 2111-2118, 2000.

[128] C. Rask-Madsen, H. Domínguez, N. Ihlemann, T. Hermann, L. Køber, and C. Torp-Pedersen, "Tumor necrosis factor- $\alpha$ inhibits insulin's stimulating effect on glucose uptake and endothelium-dependent vasodilation in humans," Circulation, vol. 108, no. 15, pp. 1815-1821, 2003.

[129] A. Picchi, X. Gao, S. Belmadani et al., "Tumor necrosis factor- $\alpha$ induces endothelial dysfunction in the prediabetic metabolic syndrome," Circulation Research, vol. 99, no. 1, pp. 69-77, 2006.

[130] A. E. Caballero, "Endothelial dysfunction in obesity and insulin resistance: a road to diabetes and heart disease," Obesity Research, vol. 11, no. 11, pp. 1278-1289, 2003.

[131] S. V. De Kreutzenberg, M. Puato, E. Kiwanuka et al., "Elevated non-esterified fatty acids impair nitric oxide independent vasodilation, in humans: evidence for a role of inwardly rectifying potassium channels," Atherosclerosis, vol. 169, no. 1, pp. 147-153, 2003.

[132] M. E. Griffin, M. J. Marcucci, G. W. Cline et al., "Free fatty acid-induced insulin resistance is associated with activation of protein kinase $\mathrm{C} \theta$ and alterations in the insulin signaling cascade," Diabetes, vol. 48, no. 6, pp. 1270-1274, 2000.

[133] H. Shi, M. V. Kokoeva, K. Inouye, I. Tzameli, H. Yin, and J. S. Flier, "TLR4 links innate immunity and fatty acid-induced insulin resistance," Journal of Clinical Investigation, vol. 116, no. 11, pp. 3015-3025, 2006.

[134] N. Houstis, E. D. Rosen, and E. S. Lander, "Reactive oxygen species have a causal role in multiple forms of insulin resistance," Nature, vol. 440, no. 7086, pp. 944-948, 2006.

[135] G. Zeng and M. J. Quon, "Insulin-stimulated production of nitric oxide is inhibited by Wortmannin: direct measurement in vascular endothelial cells," Journal of Clinical Investigation, vol. 98, no. 4, pp. 894-898, 1996.

[136] M. A. Vincent, L. H. Clerk, J. R. Lindner et al., "Microvascular recruitment is an early insulin effect that regulates skeletal muscle glucose uptake in vivo," Diabetes, vol. 53, no. 6, pp. 1418-1423, 2004. 
[137] F. H. Nystrom and M. Quon, "Insulin signalling: metabolic pathways and mechanisms for specificity," Cellular Signalling, vol. 11 , no. 8, pp. 563-574, 1999.

[138] V. Adams, A. Linke, N. Kränkel et al., "Impact of regular physical activity on the $\mathrm{NAD}(\mathrm{P}) \mathrm{H}$ oxidase and angiotensin receptor system in patients with coronary artery disease," Circulation, vol. 111, no. 5, pp. 555-562, 2005.

[139] L. K. Kelly, S. Wedgwood, R. H. Steinhorn, and S. M. Black, "Nitric oxide decreases endothelin-1 secretion through the activation of soluble guanylate cyclise," American Journal of Physiology, vol. 286, no. 5, pp. L984-L991, 2004.

[140] R. Muniyappa, M. Iantorno, and M. J. Quon, "An integrated view of insulin resistance and endothelial dysfunction," Endocrinology and Metabolism Clinics of North America, vol. 37, no. 3, pp. 685-711, 2008.

[141] A. Lteif, P. Vaishnava, A. D. Baron, and K. J. Mather, "Endothelin limits insulin action in obese/insulin-resistant humans," Diabetes, vol. 56, no. 3, pp. 728-734, 2007.

[142] G. Ahlborg, A. Shemyakin, F. Böhm, A. Gonon, and J. Pernow, "Dual endothelin receptor blockade acutely improves insulin sensitivity in obese patients with insulin resistance and coronary artery disease," Diabetes Care, vol. 30, no. 3, pp. 591-596, 2007.

[143] M. A. Vincent, M. Montagnani, and M. J. Quon, "Molecular and physiologic actions of insulin related to production of nitric oxide in vascular endothelium," Current Diabetes Reports, vol. 3, no. 4, pp. 279-288, 2003.

[144] M. Montagnani, H. Chen, V. A. Barr, and M. J. Quon, "Insulin-stimulated activation of eNOS is independent of $\mathrm{Ca}^{2+}$ but requires phosphorylation by Akt at Ser," Journal of Biological Chemistry, vol. 276, no. 32, pp. 30392-30398, 2001.

[145] F. Schinzari, M. Tesauro, V. Rovella et al., "Generalized impairment of vasodilator reactivity during hyperinsulinemia in patients with obesity-related metabolic syndrome," American Journal of Physiology, vol. 299, no. 6, pp. E947E952, 2010.

[146] V. J. Stevens, E. Obarzanek, N. R. Cook et al., "Long-term weight loss and changes in blood pressure: results of the trials of hypertension prevention, phase II," Annals of Internal Medicine, vol. 134, no. 1, pp. 1-11, 2001. 


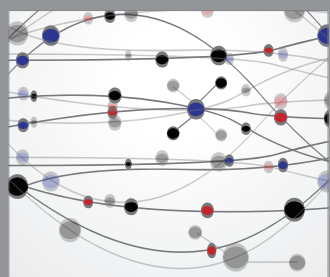

The Scientific World Journal
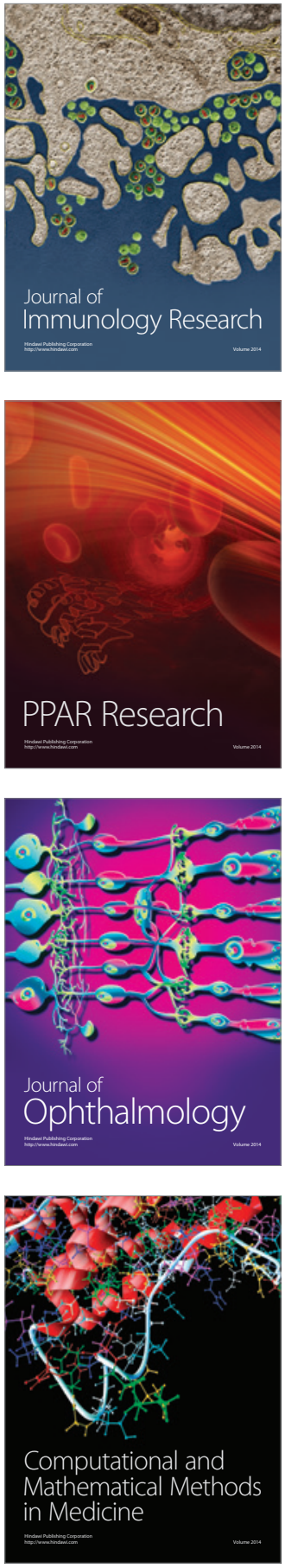

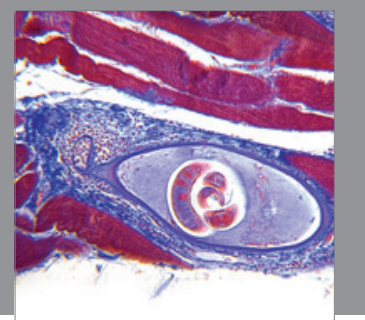

Gastroenterology

Research and Practice
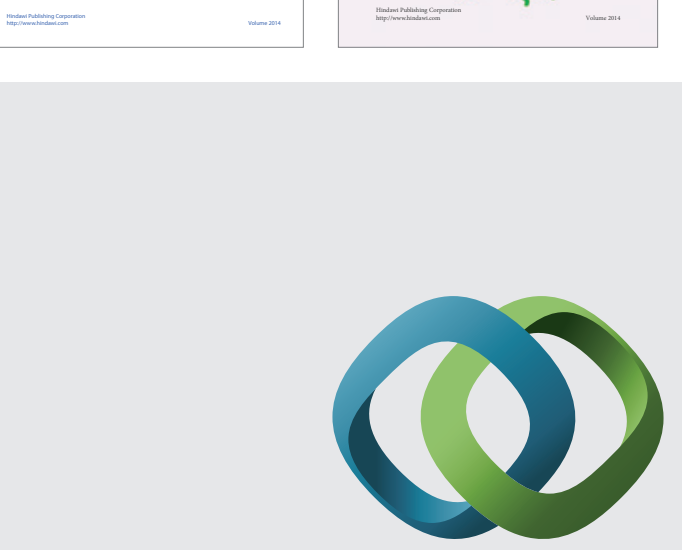

\section{Hindawi}

Submit your manuscripts at

http://www.hindawi.com
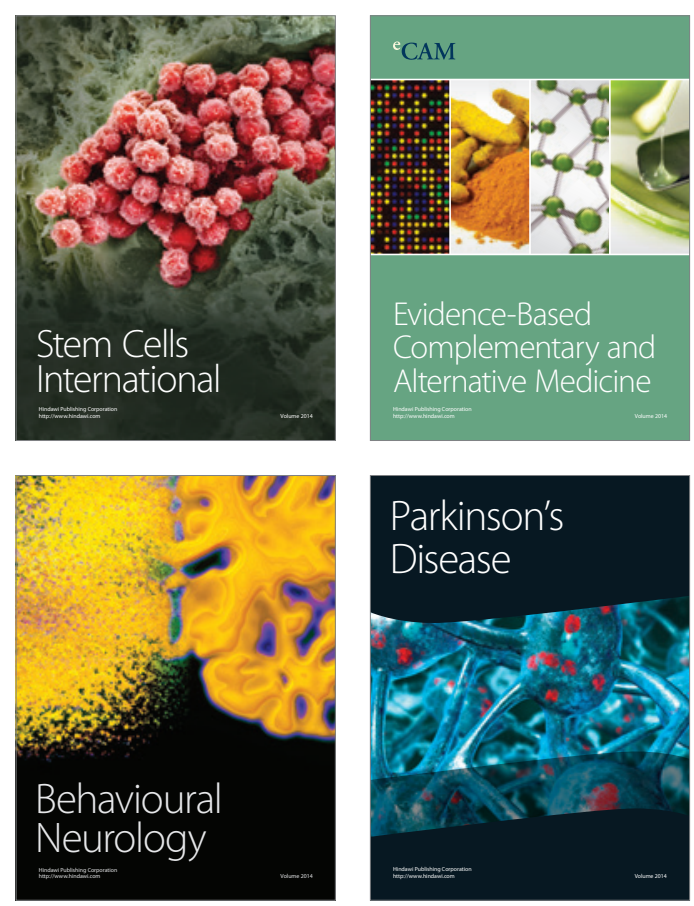

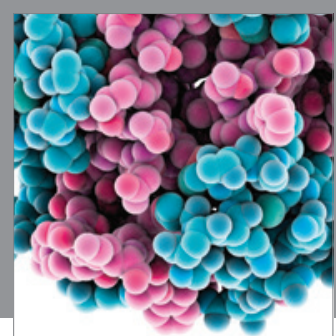

Journal of
Diabetes Research

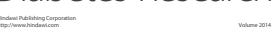

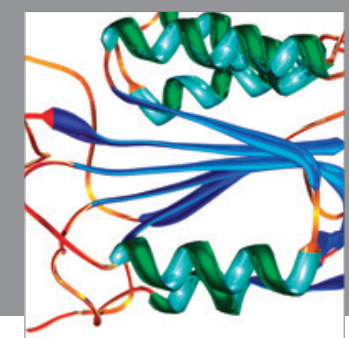

Disease Markers
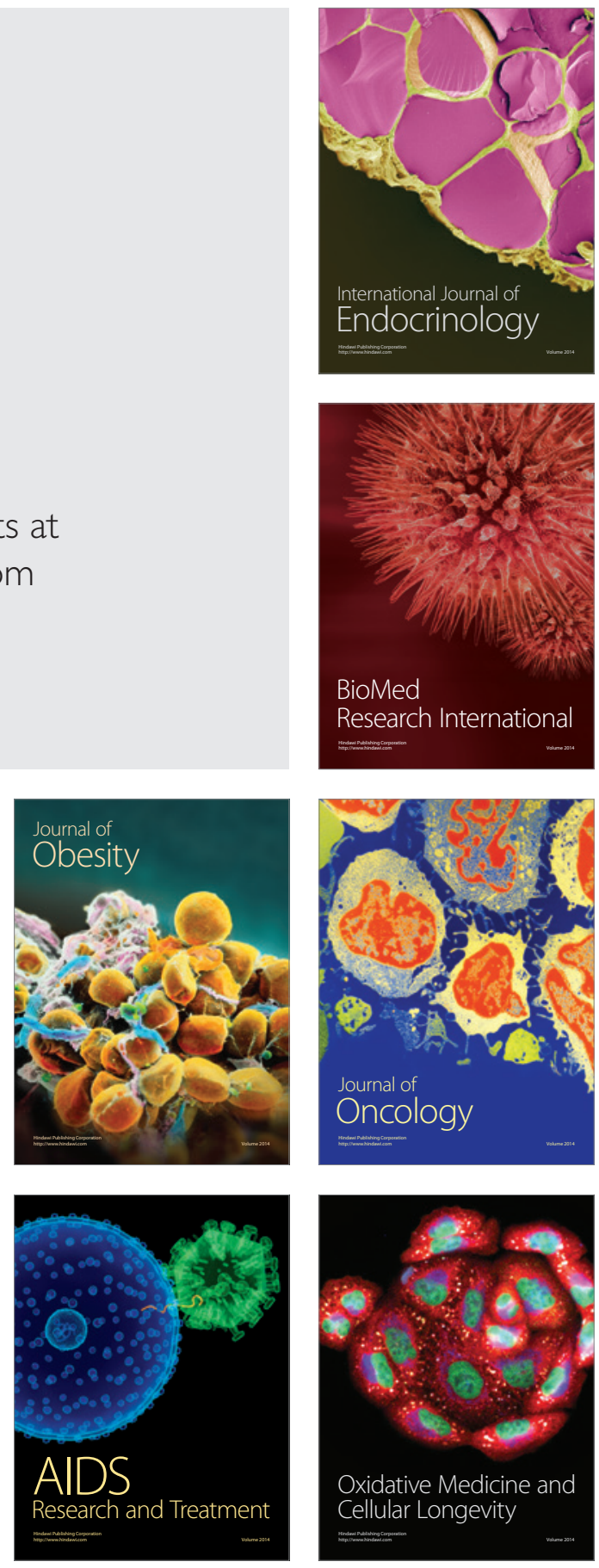